\title{
RATIONAL HOMOTOPY OF THE SPACE OF SELF-MAPS OF COMPLEXES WITH FINITELY MANY HOMOTOPY GROUPS
}

\author{
SAMUEL B. SMITH
}

\begin{abstract}
For simply connected CW complexes $X$ with finitely many, finitely generated homotopy groups, ${ }^{1}$ the path components of the function space $M(X, X)$ of free self-maps of $X$ are all of the same rational homotopy type if and only if all the $k$-invariants of $X$ are of finite order. In case $X$ is rationally a two-stage Postnikov system the space $M_{0}(X, X)$ of inessential self-maps of $X$ has the structure of rational $H$-space if and only if the $k$-invariants of $X$ are of finite order.
\end{abstract}

\section{INTRODUCTION}

Given spaces $X$ and $Y$ let $M(X, Y)$ denote the space of free, continuous maps from $X$ to $Y$ with the compact-open topology. Given a map $f: X \rightarrow Y$, we let $M_{f}(X, Y)$ denote the component of $f$ in $M(X, Y)$. In particular, we denote by $M_{0}(X, Y)$ the space of inessential maps from $X$ to $Y$ and by $M_{1}(X, X)$ the space of self-maps of $X$ which are homotopic to the identity. If $X$ and $Y$ are spaces with base points then we have the subspace $M(X, Y)_{*}$ of $M(X, Y)$ consisting of all based maps between $X$ and $Y$. Given a based map $f: X \rightarrow Y$, we write $M_{f}(X, Y)_{*}$ for the component of $f$ in $M(X, Y)_{*}$.

In his 1956 paper [20], $\mathrm{R}$. Thom used a Postnikov decomposition of $Y$ to show that the homotopy groups of $M(X, Y)$ are determined up to a series of group extensions involving the cohomology of $X$ with coefficients in the homotopy of $Y$. To illustrate his technique, Thom computed the rational homotopy groups of certain function spaces. He also showed how by analyzing the evaluation map it is possible, in certain cases, to determine the rational homotopy type of components of $M(X, Y)$. While predating by many years Sullivan's development of the theory of minimal models, Thom's work established the techniques for studying function spaces within the framework of rational homotopy theory. In [7], Haefliger completed a program initiated by Sullivan [18] with the construction of a rational homotopy-theoretic model for the space of sections of a nilpotent bundle. The construction embodied the two aspects

Received by the editors September 30, 1992.

1991 Mathematics Subject Classification. Primary 55P62, 55P15, 58D99.

Key words and phrases. Function spaces, minimal model, Postnikov tower, rational homotopy equivalence, Hirsch lemma.

${ }^{1}$ Here and throughout, this hypothesis may be replaced with the assumption that $X$ is a simply connected finite $\mathrm{CW}$ complex with finite-dimensional rational homotopy. See the remark after Proposition 2.1. 
of Thom's approach to the study of function spaces-Haefliger and Sullivan's algebraic model mirrored Thom's geometric use of Postnikov decompositions and, as Thom's work suggested, a key element of the construction was the algebraic characterization of the relevant evaluation maps. In this paper we apply Thom's methods within the context of Sullivan's minimal models to study the rational homotopy types represented by the components of function spaces.

A basic problem concerning the function space $M(X, Y)$ is to classify its components up to homotopy type. In case $X$ is an $n$-manifold and $Y$ an $n$-sphere, Hansen [8] classifies the components in terms of the degree of the map from $X$ to $Y$. Møller obtains an analogous classification theorem in [14] when $X$ and $Y$ are taken to be complex projective spaces. If $Y$ is an $H$-space with homotopy inverses, it is easy to show that the components of $M(X, Y)$ represent one homotopy type. Our first main result states that when $X=Y$ the assumption that $X$ is a rational $H$-space is necessary to ensure the components of $M(X, X)$ are of one rational homotopy type. We prove

Theorem 1. If $X$ is a simply connected complex with finitely many, finitely generated homotopy groups, the path components of $M(X, X)$ are all of the same rational homotopy type if and only if the $k$-invariants of $X$ are all of finite order.

The proof of Theorem 1 is computational; we use rational Postnikov towers together with Thom's method for calculating homotopy groups of function spaces, which we recall in $\S 3$. In fact, the key lemma (Lemma 3.2) is the calculation of the rational homotopy groups of the space $M_{0}(X, Y)$ for $X$ and $Y$ simply connected spaces of finite homotopy type. Our computational method also permits the determination of the rational homotopy type of the space $M_{1}(X, X)$ for certain spaces $X$, which has implications for the theory of classifying fibrations (see $\S 5)$. As an example we determine the rational homotopy type of $M_{1}(X, X)$ when $X$ is a product of spheres (Theorem 2).

If $X$ is an $H$-space then multiplication of maps gives the spaces $M_{0}(X, X)_{*}$ and $M_{0}(X, X)$ the structure of $H$-space also. In Lemma 6.1, we prove that if $X$ is a two-stage Postnikov system, then the space of based inessential maps $M_{0}(X, X)_{*}$ has the structure of $H$-space regardless of whether or not $X$ does. This fact contrasts sharply with the situation for the space of free inessential maps. In fact, our second main result implies that if $X$ is rationally a twostage Postnikov system, then the assumption that $X$ is a rational $H$-space is necessary to ensure that $M_{0}(X, X)$ has the structure of rational $H$-space. We prove

Theorem 3. Let $X$ be a simply connected complex with finitely many, finitely generated homotopy groups whose rationalization is a two-stage Postnikov system. Then there is a rational equivalence

$$
M_{0}(X, X) \simeq_{\mathbb{Q}} X \times M_{0}(X, X)_{*} .
$$

The proof of Theorem 3 consists in constructing the minimal model for $M_{0}(X, X)$. To accomplish this we use the Hirsch Lemma [4, Lemma 3.1] and the algebraic characterization of certain evaluation maps given by Haefliger [7], which we recall in $\S 7$. Our proof follows the approach taken by Møller and Raussen in [15]. We remark that since $M_{1}(X, X)$ always has the structure of 
$H$-space, with multiplication given by composition of maps, Theorem 3 can be viewed as a refinement of Theorem 1 for two-stage Postnikov systems.

This paper represents a portion of the author's Ph.D. thesis. The author would like to thank his thesis advisor, Professor Donald Kahn, for his assistance and encouragement during the preparation of this work.

\section{Preliminaries}

In this section we recall results and establish notation for use in the sequel. We begin with a result on

Function spaces and rationalization. The space $M(X, Y)$ does not, in general, have the homotopy type of a CW complex. (For an example of this failure, see [13, p. 273].) Since we wish to consider the rationalization of components of $M(X, Y)$, we must restrict the spaces $X$ and $Y$ under consideration so that the components of $M(X, Y)$ are nilpotent $\mathrm{CW}$ complexes. The following proposition provides us with a suitable class of spaces with which to work:

Proposition 2.1. Let $X$ and $Y$ be simply connected countable $C W$ complexes with either

(a) $X$ a finite complex or

(b) $X$ having finitely generated homotopy groups and $Y$ having finitely many, finitely generated homotopy groups.

Then each component $M_{f}(X, Y)$ of $M(X, Y)$ is a nilpotent $C W$ complex. Moreover, if $e: Y \rightarrow Y_{0}$ is the rationalization of $Y$, then the induced map $\underline{e}: M_{f}(X, Y) \rightarrow M_{e \circ f}\left(X, Y_{0}\right)$ obtained by composition with $e$, is the rationalization of $M_{f}(X, Y)$. The analogous result holds on based mapping spaces.

Proof. When $X$ is a finite complex, Milnor [13] shows that $M_{f}(X, Y)$ has the homotopy type of a (countable) complex. The result on the nilpotence and the rationalization of $M_{f}(X, Y)$ in this case is [10, Theorem 2.5]. In case (b) the fact that $M_{f}(X, Y)$ has the homotopy type of a complex is due to P. Kahn [12]. For nilpotence, we observe that the proof of [11, Theorem A] goes through if $Y$ is a finite Postnikov piece (as opposed to being homologically finite). Similarly, the result on the rationalization of $M_{f}(X, Y)$ in case (b) can be deduced from [11, Theorem B].

In view of the proposition, we state our results in terms of simply connected complexes with finitely many, finitely generated homotopy groups. We remark, however, that we could work equally well with simply connected finite complexes $X$ with finite-dimensional rational homotopy (i.e., $\sum_{n \geq 2} \operatorname{dim}_{\mathbb{Q}} \pi_{n}(X) \otimes \mathbb{Q}<$ $\infty)$

Minimal models. We next establish notation for working with differential graded algebras $(D G A \mathrm{~s})$ and Sullivan's theory of minimal models. Our references for rational homotopy theory are $[2,4,18,19]$.

By a DGA, $\left(\mathscr{A}^{*}, d\right)$, we mean a connected commutative graded algebra $\mathscr{A}^{*}$ over $\mathbb{Q}$ together with an algebra derivation $d$ of degree one whose square is zero. Given a rational vector space $V$, we denote by $\Lambda_{n}(V)$ the free 
graded algebra generated in degree $n>0$ by the elements of $V$. Alternately, if $x_{1}, \ldots, x_{l}$ are a basis for $V$ we may write $\Lambda_{n}\left(x_{1}, \ldots, x_{l}\right)$ for $\Lambda_{n}(V)$. A graded algebra $\mathscr{B}^{*}$ is free if $\mathscr{B}^{*}$ may be written in the form $\mathscr{B}^{*}=$ $\bigotimes_{n=1}^{\infty} \Lambda_{n}\left(V_{n}\right)$. In this case, we will write $\operatorname{Vect}_{n}\left(\mathscr{B}^{*}\right)=V_{n}$ for the vector space of degree $n$ generators of $\mathscr{B}^{*}$.

A DGA $\left(\mathscr{B}^{*}, d\right)$ is minimal if $\mathscr{B}^{*}$ is free as graded algebra, $\mathscr{B}^{*}=$ $\bigotimes_{n=1}^{\infty} \Lambda_{n}\left(V_{n}\right)$, where the graded vector space $\bigoplus_{n=1}^{\infty} V_{n}$ admits a well-ordered, homogeneous basis $x_{\alpha}$ such that $d x_{\alpha}$ is a polynomial without linear term in the $x_{\beta}$ with $\beta<\alpha$. In particular, if $\mathscr{B}^{*}$ is free and simply connected then $\left(\mathscr{B}^{*}, d\right)$ is minimal if and only if the image of $d$ is contained in the decomposables of $\mathscr{B}^{*}$.

If $\left(\mathscr{A}^{*}, d\right)$ is a DGA and $\mathscr{B}^{*}$ is a free graded algebra given as above, then we may form a new DGA $\left(\mathscr{C}^{*}, d^{\prime}\right)$, which we denote by $\mathscr{C}^{*}=\mathscr{A}^{*} \otimes_{d^{\prime}} \mathscr{B}^{*}$, where, as graded algebra, $\mathscr{C}^{*}=\mathscr{A}^{*} \otimes \mathscr{B}^{*}$, and where the differential $d^{\prime}$ is defined by $d^{\prime} \mid \mathscr{A}^{*}=d$, while $d^{\prime}$ on $\mathscr{B}^{*}$ is some degree one derivation from $\mathscr{B}^{*}$ to the cocycles of $\left(\mathscr{A}^{*}, d\right)$. Note that defining $d^{\prime}$ is equivalent to defining linear maps $d^{\prime}: V_{n} \rightarrow H^{n+1}\left(\mathscr{A}^{*}, d\right)$.

If $X$ is a nilpotent $\mathrm{CW}$ complex, then we have the de Rham-Sullivan DGA $\left(\Omega^{*}(X), \delta_{X}\right)$ which has the property that $H\left(\Omega^{*}(X), \delta_{X}\right) \cong H^{*}(X, \mathbb{Q})$ by an isomorphism which is induced by a chain map to the rational cochains of $X$. We say $\left(\mathscr{A}^{*}(X), d_{X}\right)$ is a model for $X$ if there is a chain map from $\left(\mathscr{A}^{*}(X), d_{X}\right)$ to the de Rham-Sullivan DGA which induces an isomorphism on cohomology. A model $\left(\mathscr{A}^{*}(X), d_{X}\right)$ is a minimal model for $X$ if $\left(\mathscr{A}^{*}(X), d_{X}\right)$ is a minimal DGA. Sullivan [18] proves the existence and uniqueness (up to DGA isomorphism) of a minimal model for $X$ as hypothesized. He shows, moreover, that the minimal model of $X$ is, in fact, a unique invariant of the rational homotopy type of $X$. We will write $\left(\mathscr{M}^{*}(X), d_{X}\right)$ for the minimal model of $X$.

\section{Results of Thom AND a calculation}

We begin this section by recalling results of Thom in [20]. Let $G$ be any abelian group and $n \geq 1$. Then, for any complex $X$, Thom observes that the components of $M(X, K(G, n))$ represent a single homotopy type. Since $K(G, n)$ has the structure of abelian topological group so does $M_{0}(X, K(G, n))$. Therefore, $M_{0}(X, K(G, n))$ has the homotopy type of a product of EilenbergMac Lane spaces. Thom constructs an isomorphism

$$
\pi_{p}\left(M_{0}(X, K(G, n))\right) \cong H^{n-p}(X, G),
$$

which is defined as follows. Given $\alpha \in \pi_{p}\left(M_{0}(X, K(G, n))\right)$, by the exponential law $\alpha$ is represented by a map $F: S^{p} \times X \rightarrow K(G, n)$ with $F_{\mid * \times X}=*$. Let $l_{n} \in H^{n}(K(G, n), G)$ be the fundamental class. Then, since $F_{\mid * \times X}=*$, we may write $F^{*}\left(l_{n}\right)=s_{p} \otimes a_{n-p} \in H^{n}\left(S^{p} \times X, G\right)$ for some $a_{n-p} \in H^{n-p}(X, G)$ where $s_{p} \in H^{p}\left(S^{p}, \mathbb{Z}\right)$ is a fixed generator. The isomorphism sends $\alpha$ to $a_{n-p}$.

Given a fibration $q: E \rightarrow B$ and any map $f: X \rightarrow E$, we have a fibration $\underline{q}: M_{f}(X, E) \rightarrow M_{q \circ f}(X, B)$, where $\underline{q}$ is the map defined by composition with $q$. Thom argues that if $q$ is a principal fibration with fibre $F=K(G, n)$, then a component of the fibre of $\underline{q}$ may be identified as $M_{0}(X, K(G, n))$. Thus 
for principal fibrations $K(G, n) \hookrightarrow E \stackrel{q}{\rightarrow} B$ we have a fibre sequence on free mapping spaces of the form

$$
M_{0}(X, K(G, n)) \rightarrow M_{f}(X, E) \stackrel{q}{\rightarrow} M_{q \circ f}(X, B) .
$$

Corresponding to this fibre sequence we have a long exact sequence on homotopy of the form

$$
\cdots \rightarrow \pi_{p}\left(M_{f}(X, E)\right) \rightarrow \pi_{p}\left(M_{q \circ f}(X, B)\right) \stackrel{\partial}{\rightarrow} \pi_{p-1}\left(M_{0}(X, K(G, n))\right) \rightarrow \cdots
$$

As regards the boundary homomorphism $\partial$ in this sequence, Thom gives the following characterization which takes into account the identification of $\pi_{p-1}\left(M_{0}(X, K(G, n))\right)$ with $H^{n+1-p}(X, G)$.

Let $k_{n+1} \in H^{n+1}(B, G)$ be the $k$-invariant for the principal fibration

$$
K(G, n) \hookrightarrow E \stackrel{q}{\rightarrow} B
$$

Then, given $F: S^{p} \times X \rightarrow B$ with $F_{\mid * \times X}=q \circ f$ representing $\alpha \in$ $\pi_{p}\left(M_{q \circ f}(X, B)\right)$, we have that $F^{*}\left(k_{n+1}\right) \in H^{n+1}\left(S^{p} \times X, G\right)$ and, as such, we may write

$$
F^{*}\left(k_{n+1}\right)=s_{p} \otimes a_{n+1-p}+1 \otimes a_{n+1} .
$$

However, since $F_{\mid * \times X}$ admits a lifting to $E$ we see that $\left(F_{\mid * \times X}\right)^{*}\left(k_{n+1}\right)=0$ so that actually

$$
F^{*}\left(k_{n+1}\right)=s_{p} \otimes a_{n+1-p} \in H^{n+1}\left(S^{p} \times X, G\right),
$$

for some $a_{n+1-p} \in H^{n+1-p}(X, G)$. Thom argues that the image of $\alpha \in$ $\pi_{p}\left(M_{q \circ f}(X, B)\right)$ under the boundary homomorphism

$$
\partial: \pi_{p}\left(M_{q \circ f}(X, B) \rightarrow \pi_{p-1}\left(M_{0}(X, K(G, n))\right) \cong H^{n+1-p}(X, G)\right.
$$

identified in $H^{n+1-p}(X, G)$ is the cohomology class $a_{n+1-p}$. We use these results to prove

Lemma 3.2. Let $X$ and $Y$ be simply connected complexes with finitely many, finitely generated homotopy groups. Let $V_{n}=\pi_{n}(Y) \otimes \mathbb{Q}, \quad n>1$. Choose $N$ such that $V_{n}=0$ for $n>N$. Then, the rational homotopy groups of the free mapping space $M_{0}(X, Y)$ are given, for $p>0, b y$

$$
\pi_{p}\left(M_{0}(X, Y)\right) \otimes \mathbb{Q} \cong \bigoplus_{n=2}^{N} H^{n-p}\left(X, V_{n}\right) .
$$

Proof. Let $Y_{n}, n=2, \ldots, N$, be the terms in the Postnikov decomposition of the rationalization $Y_{0}$ of $Y$. Then for each $n=3, \ldots, N$, we have a principal fibration $p_{n}: Y_{n} \rightarrow Y_{n-1}$ with fibre $K\left(V_{n}, n\right)$, and we may assume that $Y_{N}=Y_{0}$. By the above, we have fibre sequences

$$
M_{0}\left(X, K\left(V_{n}, n\right)\right) \rightarrow M_{0}\left(X, Y_{n}\right) \stackrel{p_{n}}{\longrightarrow} M_{0}\left(X, Y_{n-1}\right) .
$$

Our method will be to show that, for each such fibre sequence, the boundary homomorphism

$$
\partial: \pi_{p}\left(M_{0}\left(X, Y_{n-1}\right)\right) \rightarrow \pi_{p-1}\left(M_{0}\left(X, K\left(V_{n}, n\right)\right)\right)
$$

is zero for all $p>0$. 
Before proving this fact let us observe that it implies the result. By Proposition 2.1 and our assumptions on $X$ and $Y$, we have, regarding the rationalization $\left(M_{0}(X, Y)\right)_{0}$ of $M_{0}(X, Y)$, that

$$
\left(M_{0}(X, Y)\right)_{0} \simeq M_{0}\left(X, Y_{0}\right)=M_{0}\left(X, Y_{N}\right) .
$$

If all boundary homomorphisms vanish, as will be shown, then, from the exact homotopy sequences corresponding to the above fibrations, we see inductively that

$$
\begin{aligned}
\pi_{p}\left(M_{0}(X, Y)\right) \otimes \mathbb{Q}= & \pi_{p}\left(M_{0}\left(X, Y_{N}\right)\right) \\
\cong & \pi_{p}\left(M_{0}\left(X, Y_{N-1}\right)\right) \oplus \pi_{p}\left(M_{0}\left(X, K\left(V_{N}, N\right)\right)\right) \\
\cong & \pi_{p}\left(M_{0}\left(X, Y_{N-2}\right)\right) \oplus \pi_{p}\left(M_{0}\left(X, K\left(V_{N-1}, N-1\right)\right)\right) \\
& \oplus \pi_{p}\left(M_{0}\left(X, K\left(V_{N}, N\right)\right)\right) \\
\cong & \cdots \cong \bigoplus_{n=2}^{N} \pi_{p}\left(M_{0}\left(X, K\left(V_{n}, n\right)\right)\right) .
\end{aligned}
$$

To recover the statement of the lemma, we use Thom's result (3.1) that

$$
\pi_{p}\left(M_{0}\left(X, K\left(V_{n}, n\right)\right)\right) \cong H^{n-p}\left(X, V_{n}\right) .
$$

To show the boundary homomorphism

$$
\partial: \pi_{p}\left(M_{0}\left(X, Y_{n-1}\right)\right) \rightarrow \pi_{p-1}\left(M_{0}\left(X, K\left(V_{n}, n\right)\right)\right)
$$

is zero we use the characterization given it by Thom, recalled above.

Let $\alpha \in \pi_{p}\left(M_{0}\left(X, Y_{n-1}\right)\right)$. Choose $F: S^{p} \times X \rightarrow Y_{n-1}$ with $F_{\mid * \times X}=*$ representing $\alpha$. Let $k_{n+1} \in H^{n+1}\left(Y_{n-1}, V_{n}\right)$ be the $k$-invariant of the principal fibration $K\left(V_{n}, n\right) \hookrightarrow Y_{n} \stackrel{p_{n}}{\rightarrow} Y_{n-1}$. Then we have that

$$
\partial(\alpha) \in \pi_{p-1}\left(M_{0}\left(X, K\left(V_{n}, n\right)\right)\right)
$$

is identified as $a_{n+1-p} \in H^{n+1-p}\left(X, V_{n}\right)$ where

$$
F^{*}\left(k_{n+1}\right)=s_{p} \otimes a_{n+1-p} \in H^{n+1}\left(S^{p} \times X, V_{n}\right) .
$$

To show $\partial(\alpha)=0$ then it suffices to show

$$
F^{*}: H^{n+1}\left(Y_{n-1}, \mathbb{Q}\right) \rightarrow H^{n+1}\left(S^{p} \times X, \mathbb{Q}\right)
$$

is zero. We prove the latter fact by considering the DGA map

$$
\mathscr{M}^{*}(F):\left(\mathscr{M}^{*}\left(Y_{n-1}\right), d_{Y_{n-1}}\right) \rightarrow\left(\mathscr{M}^{*}\left(S^{p} \times X\right), d_{S^{p} \times X}\right)
$$

induced on minimal models by $F: S^{p} \times X \rightarrow Y_{n-1}$.

By the Künneth formula for minimal models $[19$, p. 75$]$ the minimal model of $S^{p} \times X$ is given as

$$
\left(\mathscr{M}^{*}\left(S^{p} \times X\right), d_{S^{p} \times X}\right) \cong\left(\mathscr{M}^{*}\left(S^{p}\right) \otimes \mathscr{M}^{*}(X), d_{S^{p}} \otimes d_{X}\right) .
$$

Recall that $\left(\mathscr{K}^{*}\left(S^{p}\right), d_{S^{p}}\right)$ is given by

$$
\left(\mathscr{K}^{*}\left(S^{p}\right), d_{S^{p}}\right)=\left\{\begin{array}{l}
\Lambda_{p}\left(x_{p}\right), \quad d_{S^{p}}=0 \text { for } p \text { odd } \\
\Lambda_{p}\left(x_{p}\right) \otimes_{d_{S^{p}}} \Lambda_{2 p-1}\left(y_{2 p-1}\right), \quad d_{S^{p}}\left(y_{2 p-1}\right)=x_{p}^{2} \text { for } p \text { even. }
\end{array}\right.
$$


Thus for $p$ odd or even, $\mathscr{M}^{*}\left(S^{p} \times X\right)$ decomposes as graded vector space in the form

$$
\mathscr{M}^{*}\left(S^{p} \times X\right)=\left(1 \otimes \mathscr{M}^{*}(X)\right) \oplus\left(x_{p} \otimes \mathscr{M}^{*}(X)\right) \oplus\left(\bigoplus_{i \neq 0, p} \mathscr{M}^{i}\left(S^{p}\right) \otimes \mathscr{M}^{*}(X)\right),
$$

where we write $\mathscr{M}^{i}\left(S^{p}\right)$ for the elements of degree $i$ in $\mathscr{M}^{*}\left(S^{p}\right)$. Put $\mathscr{A}^{*}=$ $\bigoplus_{i \neq 0, p} \mathscr{M}^{i}\left(S^{p}\right) \otimes \mathscr{M}^{*}(X)$, and observe that $\mathscr{A}^{*}$ is, in fact, a sub-DGA (albeit without unit) of $\mathscr{M}^{*}\left(S^{p} \times X\right)$. We denote the restriction of $d_{S^{p} \times X}$ to $\mathscr{A}^{*}$ by $d_{\mathscr{A}}$.

Since $Y_{n-1}$ is simply connected and $n$-coconnected (i.e., $\pi_{q}\left(Y_{n-1}\right)=0$ for $q \geq n$ ) by the Sullivan isomorphism [2, p. 65], the minimal model for $Y_{n-1}$ is generated by elements of degree $<n$. Thus $\mathscr{M}^{n+1}\left(Y_{n-1}\right)$ is additively generated by decomposable elements. Let $z_{n+1} \in \mathscr{M}^{n+1}\left(Y_{n-1}\right)$ be of the form $z_{n+1}=$ $\prod_{i} z_{i}$ where $z_{i} \in \mathscr{M}^{r_{i}}\left(Y_{n-1}\right)$ and $0<r_{i}<n$. Then

$$
\mathscr{M}^{*}(F)\left(z_{n+1}\right)=\mathscr{M}^{*}(F)\left(\prod_{i} z_{i}\right)=\prod_{i} \mathscr{M}^{*}(F)\left(z_{i}\right) \in \mathscr{M}^{n+1}\left(S^{p} \times X\right) .
$$

Using (3.3) we may write $\mathscr{M}^{*}(F)\left(z_{i}\right)$ in the form

$$
\mathscr{M}^{*}(F)\left(z_{i}\right)=1 \otimes a_{i}+x_{p} \otimes b_{i}+C_{i}
$$

where $a_{i} \in \mathscr{M}^{r_{i}}(X), \quad b_{i} \in \mathscr{M}^{r_{i}-p}(X)$, and $C_{i} \in \mathscr{A}^{r_{i}}$. But now, since $F_{\mid * \times X}=$ $*$, we see that each $a_{i}=0$. We conclude that actually

$$
\mathscr{M}^{*}(F)\left(z_{n+1}\right)=\prod_{i} \mathscr{M}^{*}(F)\left(z_{i}\right)=\prod_{i}\left(x_{p} \otimes b_{i}+C_{i}\right) \in \mathscr{A}^{n+1} .
$$

Since $\mathscr{M}^{n+1}\left(Y_{n-1}\right)$ is additively generated by such decomposables $z_{n+1}$, we have shown that $\mathscr{M}^{*}(F)$ maps $\mathscr{M}^{n+1}\left(Y_{n-1}\right)$ into $\mathscr{A}^{n+1}$.

Now, since $H^{i}\left(S^{p}, \mathbb{Q}\right)=0$ for $i \neq 0, p$, the DGA $\left(\mathscr{A}^{*}, d_{\mathscr{A}}\right)$ is, by its definition, acyclic; i.e., $H^{*}\left(\mathscr{A}^{*}, d_{\mathscr{A}}\right)=0$. As a result of the preceding paragraph then, the map induced by $\mathscr{M}^{*}(F)$ on degree $n+1$ cohomology is zero. Finally, recall that the map induced by $\mathscr{M}^{*}(F)$ on degree $n+1$ cohomology is just $F^{*}: H^{n+1}\left(Y_{n-1}, \mathbb{Q}\right) \rightarrow H^{n+1}\left(S^{p} \times X, \mathbb{Q}\right)$, and the proof is complete.

Remark. Lemma 3.2 asserts that the differentials in the Federer spectral sequence [6] $E_{i j}^{2}=H^{-i}\left(X, \pi_{j}(Y)\right) \Rightarrow \pi_{i+j}\left(M_{0}(X, Y)\right)$ have finite order. The referee has pointed out that this fact is well-known and easy to prove when $X$ and $Y$ are spectra and the spectral sequence is the Atiyah-Hirzebruch spectral sequence.

\section{Proof of Theorem 1}

The proof of Theorem 1 consists in comparing the rational homotopy groups of $M_{0}(X, X)$ with those of $M_{1}(X, X)$. In preparation for the proof, we follow Thom's approach to obtain a characterization (Lemma 4.4) of the boundary homomorphisms in the long exact homotopy sequence of certain mapping space fibrations. 
For $l=2, \ldots, n+1$, let $V_{l}$ be a rational vector space and let $X_{n}=$ $\prod_{l=2}^{n} K\left(V_{l}, l\right)$. Let $K\left(V_{n+1}, n+1\right) \hookrightarrow X_{n+1} \stackrel{p_{n+1}}{\longrightarrow} X_{n}$ be a principal fibration with classifying map $k_{n+2}: X_{n} \rightarrow K\left(V_{n+1}, n+2\right)$. Given a space $X$ and any map $f_{n+1}: X \rightarrow X_{n+1}$ let $f_{n}=p_{n+1} \circ f_{n+1}: X \rightarrow X_{n}$. We seek to characterize the boundary homomorphisms

$$
\partial: \pi_{p}\left(M_{f_{n}}\left(X, X_{n}\right)\right) \rightarrow \pi_{p-1}\left(M_{0}\left(X, K\left(V_{n+1}, n+1\right)\right)\right)
$$

of the long exact sequence on homotopy corresponding to the fibre sequence

$$
M_{0}\left(X, K\left(V_{n+1}, n+1\right)\right) \rightarrow M_{f_{n+1}}\left(X, X_{n+1}\right) \rightarrow M_{f_{n}}\left(X, X_{n}\right) .
$$

Since $X_{n}$ is a product of Eilenberg-Mac Lane spaces, from Thom's result (3.1) we have

$$
\pi_{p}\left(M_{f_{n}}\left(X, X_{n}\right)\right) \cong \bigoplus_{l=2}^{n} H^{l-p}\left(X, V_{l}\right) \cong \bigoplus_{l=2}^{n} H^{l-p}(X, \mathbb{Q}) \otimes V_{l}^{*},
$$

where $V_{l}^{*}=\operatorname{Hom}\left(V_{l}, \mathbb{Q}\right)$. Similarly,

$$
\pi_{p-1}\left(M_{0}\left(X, K\left(V_{n+1}, n+1\right)\right)\right) \cong H^{n-p+2}(X, \mathbb{Q}) \otimes V_{n+1}^{*} .
$$

Using these identifications, we view $\partial$ in (4.1) as a map

$$
\partial: \bigoplus_{l=2}^{n} H^{l-p}(X, \mathbb{Q}) \otimes V_{l}^{*} \rightarrow H^{n-p+2}(X, \mathbb{Q}) \otimes V_{n+1}^{*} .
$$

Let $d_{l}=\operatorname{dim}_{\mathbb{Q}} V_{l}^{*}, \quad l=2, \ldots, n+1$, and let $x_{i l}, i=1, \ldots, d_{l}$, be a basis for $V_{l}^{*}$. Now $k_{n+2}$ induces a linear map $k_{n+2}^{*}: V_{n+1}^{*} \rightarrow H^{n+2}\left(X_{n}, \mathbb{Q}\right)$ on rational cohomology. Observe that $H^{*}\left(X_{n}, \mathbb{Q}\right)$ is the free graded algebra given by $H^{*}\left(X_{n}, \mathbb{Q}\right)=\bigotimes_{l=2}^{n} \Lambda_{l}\left(V_{l}^{*}\right)$. Thus the elements of $H^{n+2}\left(X_{n}, \mathbb{Q}\right)$ may be viewed as polynomials in the variables $x_{i l}, 2 \leq l \leq n$. Let $p_{j}=k_{n+2}^{*}\left(x_{j, n+1}\right) \in$ $H^{n+2}\left(X_{n}, \mathbb{Q}\right)$ be the corresponding polynomials in $x_{i l}, \quad 2 \leq l \leq n$. Then, regarding $\partial$ in (4.3), we have

Lemma 4.4. Given $a_{l_{0}-p} \in H^{l_{0}-p}(X, \mathbb{Q})$ with $2 \leq l_{0} \leq n$,

$$
\partial\left(a_{l_{0}-p} \otimes x_{i_{0} l_{0}}\right)=\sum_{j=1}^{d_{n+1}} a_{l_{0}-p} \cdot f_{n}^{*}\left(\frac{\partial p_{j}}{\partial x_{i_{0} l_{0}}}\right) \otimes x_{j, n+1} \in H^{n-p+2}(X, \mathbb{Q}) \otimes V_{n+1}^{*},
$$

for $1 \leq i_{0} \leq d_{l_{0}}$.

Proof. Let $p_{l}: X_{n} \rightarrow K\left(V_{l}, l\right)$ be the projection onto the $l$ th factor of $X_{n}$, $l=2, \ldots, n$. Define $F: S^{p} \times X \rightarrow X_{n}$ by requiring that

$\left(p_{l} \circ F\right)^{*}\left(x_{i l}\right)=\left\{\begin{array}{l}1 \otimes \bar{x}_{i_{0} l_{0}}+s_{p} \otimes a_{l_{0}-p} \in H^{l_{0}}\left(S^{p} \times X, \mathbb{Q}\right) \quad \text { for } i=i_{0}, l=l_{0}, \\ 1 \otimes \bar{x}_{i l} \in H^{l}\left(S^{p} \times X, \mathbb{Q}\right) \quad \text { otherwise, }\end{array}\right.$

where $s_{p} \in H^{p}\left(S^{p}, \mathbb{Z}\right)$ is a fixed generator, and $\bar{x}_{i l}=f_{n}^{*}\left(x_{i l}\right) \in H^{l}(X, \mathbb{Q})$. We claim that $F$ represents an element $\alpha \in \pi_{p}\left(M_{f_{n}}\left(X, X_{n}\right)\right)$. To see this, let 
$i_{2}: X \rightarrow S^{p} \times X$ be the inclusion $i_{2}(x)=(*, x)$. Observe that for each $x_{i l}$,

$$
\left(F \circ i_{2}\right)^{*}\left(x_{i l}\right)=i_{2}^{*}\left(F^{*}\left(x_{i l}\right)\right)=i_{2}^{*}\left(1 \otimes \bar{x}_{i l}+\epsilon s_{p} \otimes a_{l_{0}-p}\right)=\bar{x}_{i l}=f_{n}^{*}\left(x_{i l}\right),
$$

where $\epsilon$ is either one or zero according to whether or not $(i, l)=\left(i_{0}, l_{0}\right)$. It follows that $F_{\mid * \times X} \simeq f_{n}: X \rightarrow X_{n}$, which implies the claim. It is easy to check that this $\alpha \in \pi_{p}\left(M_{f_{n}}\left(X, X_{n}\right)\right)$ corresponds to $a_{l_{0}-p} \otimes x_{i_{0} l_{0}} \in H^{l_{0}-p}(X, \mathbb{Q}) \otimes V_{l_{0}}^{*}$ under the identification (4.2).

According to Thom's characterization of the boundary homomorphism $(\S 3)$, to prove the lemma we must show that for $1 \leq j \leq d_{n+1}$,

$$
F^{*} \circ k_{n+2}^{*}\left(x_{j, n+1}\right)=s_{p} \otimes a_{l_{0}-p} \cdot f_{n}^{*}\left(\frac{\partial p_{j}}{\partial x_{i_{0} l_{0}}}\right) \in H^{n+2}(X, \mathbb{Q}),
$$

where we recall that $p_{j}=k_{n+2}^{*}\left(x_{j, n+1}\right) \in H^{n+2}(X, \mathbb{Q})$ is a polynomial in the $x_{i l}, \quad 2 \leq l \leq n$.

Write $p_{j}$ in the form

$$
p_{j}=\left(x_{i_{0} l_{0}}\right)^{n_{1}} m_{1}+\cdots+\left(x_{i_{0} l_{0}}\right)^{n_{t}} m_{t}+p_{j}^{\prime},
$$

where $p_{j}^{\prime}$ is a polynomial and the $m_{k}$ are monomials which do not involve the variable $x_{i_{0} l_{0}}$. Using the fact that $s_{p}^{2}=0$, we compute

$$
\begin{aligned}
& F^{*} \circ k_{n+2}^{*}\left(x_{j, n+1}\right)=F^{*}\left(p_{j}\right)=F^{*}\left(\left(x_{i_{0} l_{0}}\right)^{n_{1}} m_{1}+\cdots+\left(x_{i_{0} l_{0}}\right)^{n_{t}} m_{t}+p_{j}^{\prime}\right) \\
&=\left(1 \otimes \bar{x}_{i_{0} l_{0}}+s_{p} \otimes a_{l_{0}-p}\right)^{n_{1}}\left(1 \otimes f_{n}^{*}\left(m_{1}\right)\right) \\
&+\cdots+\left(1 \otimes \bar{x}_{i_{0} l_{0}}+s_{p} \otimes a_{l_{0}-p}\right)^{n_{t}}\left(1 \otimes f_{n}^{*}\left(m_{t}\right)\right)+1 \otimes f_{n}^{*}\left(p_{j}^{\prime}\right) \\
&= n_{1}\left(s_{p} \otimes a_{l_{0}-p}\left(\bar{x}_{i_{0} l_{0}}\right)^{n_{1}-1} f_{n}^{*}\left(m_{1}\right)\right)+\cdots+n_{t}\left(s_{p} \otimes a_{l_{0}-p}\left(\bar{x}_{i_{0} l_{0}}\right)^{n_{t}-1} f_{n}^{*}\left(m_{t}\right)\right) \\
&+1 \otimes\left(\bar{x}_{i_{0} l_{0}}\right)^{n_{1}} f_{n}^{*}\left(m_{1}\right)+\cdots+1 \otimes\left(\bar{x}_{i_{0} l_{0}}\right)^{n_{t}} f_{n}^{*}\left(m_{t}\right)+1 \otimes f_{n}^{*}\left(p_{j}^{\prime}\right) \\
&= s_{p} \otimes a_{l_{0}-p} \cdot f_{n}^{*}\left(\frac{\partial p_{j}}{\partial x_{i_{0} l_{0}}}\right)+1 \otimes f_{n}^{*}\left(p_{j}\right)=s_{p} \otimes a_{l_{0}-p} \cdot f_{n}^{*}\left(\frac{\partial p_{j}}{\partial x_{i_{0} l_{0}}}\right) .
\end{aligned}
$$

(Note : $f_{n}^{*}\left(p_{j}\right)=\left(k_{n+2} \circ f_{n}\right)^{*}\left(x_{j, n+1}\right)=0$ since $k_{n+2} \circ f_{n} \simeq *$.)

We are now prepared to prove

Theorem 1. Let $X$ be a simply connected $C W$ complex with finitely many, finitely generated homotopy groups. Then, the components of $M(X, X)$ are all of the same rational homotopy type if and only if all the $k$-invariants of $X$ are of finite order.

Proof. If the $k$-invariants of $X$ are all of finite order, then the rationalization $X_{0}$ of $X$ is a product of (rational) Eilenberg-Mac Lane spaces-a finite product since $X$ has finite-dimensional rational homotopy. Thus $X_{0}$ has the homotopy type of an abelian topological group and so the components of $M\left(X, X_{0}\right)$ are of the same homotopy type. Sufficiency now follows easily from the fact that, by Proposition 2.1, given any $f: X \rightarrow X$ the map $\underline{e}: M_{f}(X, X) \rightarrow M_{e \circ f}\left(X, X_{0}\right)$ is the rationalization of $M_{f}(X, X)$, where $e: X \rightarrow X_{0}$ is the rationalization of $X$.

To prove necessity we show that if such a complex $X$ has an infinite order $k$ invariant, then the rational homotopy groups of $M_{1}(X, X)$ are not isomorphic to those of $M_{0}(X, X)$. 
Suppose $X$ is as in the hypothesis and has an infinite order $k$-invariant. Let $\left\{X_{n}\right\}_{n=2}^{\infty}$ be the spaces in the Postnikov decomposition of the rationalization $X_{0}$ of $X$. Since $X$ has finite-dimensional rational homotopy, we may suppose that $X_{N}=X_{0}$ for some $N \gg 0$. We are given principal fibrations $p_{n}: X_{n} \rightarrow X_{n-1}$ with fibre $K\left(V_{n}, n\right)$, where $V_{n}=\pi_{n}(X) \otimes \mathbb{Q}$ and $n$-equivalences $f_{n}: X_{0} \rightarrow X_{n}$ with $p_{n} \circ f_{n}=f_{n-1}$. We take $f_{N}: X_{0} \rightarrow X_{N}=X_{0}$ to be the identity.

Let $f: X \rightarrow X$ be any map. For each $n$ we have the fibre sequence

$$
M_{0}\left(X, K\left(V_{n}, n\right)\right) \rightarrow M_{f_{n} \circ e \circ f}\left(X, X_{n}\right) \stackrel{p_{n}}{\longrightarrow} M_{f_{n-1} \circ e \circ f}\left(X, X_{n-1}\right) .
$$

Thus, for each $p>0$, corresponding to the above fibre sequence we have the exact sequence

$$
\pi_{p}\left(M_{0}\left(X, K\left(V_{n}, n\right)\right)\right) \rightarrow \pi_{p}\left(M_{f_{n} \text { oe } f}\left(X, X_{n}\right)\right) \stackrel{\left(\underline{p}_{n}\right) *}{\longrightarrow} \pi_{p}\left(M_{f_{n-1} \text { oe } f}\left(X, X_{n-1}\right)\right) .
$$

Now $X_{2}=K\left(V_{2}, 2\right)$ and

$$
M_{f_{N} \circ e \circ f}\left(X, X_{N}\right)=M_{e \circ f}\left(X, X_{0}\right) \simeq\left(M_{f}(X, X)\right)_{0} .
$$

Since, by the above sequence,

$$
\begin{aligned}
\operatorname{dim}_{\mathbb{Q}} \pi_{p}\left(M_{f_{n} \circ e \circ f}\left(X, X_{n}\right)\right) \leq & \operatorname{dim}_{\mathbb{Q}} \pi_{p}\left(M_{0}\left(X, K\left(V_{n}, n\right)\right)\right) \\
& +\operatorname{dim}_{\mathbb{Q}} \pi_{p}\left(M_{f_{n-1} \circ e \circ f}\left(X, X_{n-1}\right)\right),
\end{aligned}
$$

and since $\pi_{p}\left(M_{0}\left(X, K\left(V_{n}, n\right)\right)\right) \cong H^{n-p}\left(X, V_{n}\right)$, we see by an easy induction that, for $p>0$,

$$
\operatorname{dim}_{\mathbb{Q}} \pi_{p}\left(M_{f}(X, X)\right) \otimes \mathbb{Q} \leq \sum_{n=2}^{N} \operatorname{dim}_{\mathbb{Q}} H^{n-p}\left(X, V_{n}\right) .
$$

In Lemma 3.2 we showed that, if $f: X \rightarrow X$ is taken to be the constant map, then the inequality (4.5) is actually an equality for all $p>0$. To prove the theorem, we take $f: X \rightarrow X$ to be the identity and show that there exists $p>0$ for which the inequality (4.5) is strict. To show this, it suffices to prove that, for some $p>0$ and some $n$ in the range $2 \leq n \leq N$, the boundary homomorphism

$$
\partial: \pi_{p}\left(M_{f_{n} \circ e}\left(X, X_{n}\right)\right) \rightarrow \pi_{p-1}\left(M_{0}\left(X, K\left(V_{n+1}, n+1\right)\right)\right),
$$

corresponding to the exact sequence on homotopy of the fibration

$$
M_{0}\left(X, K\left(V_{n+1}, n+1\right)\right) \rightarrow M_{f_{n+1} \circ e}\left(X, X_{n+1}\right) \stackrel{p_{n+1}}{\longrightarrow} M_{f_{n} \circ e}\left(X, X_{n}\right)
$$

(i.e the fibration in the case where $f=1_{X}$ ) is nontrivial.

We are assuming that some $k$-invariant of $X$ is of infinite order. It follows from this that the corresponding $k$-invariant of $X_{0}$ is nontrivial. Suppose $k_{n+2} \in H^{n+2}\left(X_{n}, V_{n+1}\right)$ is the first nontrivial $k$-invariant of $X_{0}$. Then $X_{n}$ is a product of rational Eilenberg-Mac Lane spaces; in fact, $X_{n}=\prod_{l=2}^{n} K\left(V_{l}, l\right)$. With the notation of Lemma 4.4, since $k_{n+2}^{*}: V_{n+1}^{*} \rightarrow H^{n+2}\left(X_{n}, \mathbb{Q}\right)$ is nontrivial we may choose $x_{j_{0}, n+1} \in V_{n+1}^{*}$ such that $p_{j_{0}}=k_{n+2}^{*}\left(x_{j_{0}, n+1}\right) \in H^{n+2}\left(X_{n}, \mathbb{Q}\right)$ is a nonzero polynomial in the variables $x_{i l}, 2 \leq l \leq n$. Let $x_{i_{0} l_{0}}$ be a variable 
appearing in $p_{j_{0}}$. Then, according to Lemma 4.4, the boundary homomorphism

$$
\partial: \pi_{l_{0}}\left(M_{f_{n} \circ e}\left(X, X_{n}\right)\right) \rightarrow \pi_{l_{0}-1}\left(M_{0}\left(X, K\left(V_{n+1}, n+1\right)\right)\right)
$$

with identifications as in (4.3), is given on $1 \otimes x_{i_{0} l_{0}} \in H^{0}(X, \mathbb{Q}) \otimes V_{l_{0}}^{*}$ by

$$
\partial\left(1 \otimes x_{i_{0} l_{0}}\right)=\sum_{j=1}^{d_{n+1}}\left(f_{n} \circ e\right)^{*}\left(\frac{\partial p_{j}}{\partial x_{i_{0} l_{0}}}\right) \otimes x_{j, n+1} \in H^{n-l_{0}+2}(X, \mathbb{Q}) .
$$

Since $p_{j_{0}} \in H^{n+2}\left(X_{n}, \mathbb{Q}\right)$ involves the the variable $x_{i_{0} l_{0}}$, certainly $\partial p_{j_{0}} / \partial x_{i_{0} l_{0}}$ is nonzero in $H^{n-l_{0}+2}\left(X_{n}, \mathbb{Q}\right)$. Since $f_{n}$ is an $n$-equivalence and $e$ is a rational equivalence, $\left(f_{n} \circ e\right)^{*}\left(\partial p_{j_{0}} / \partial x_{i_{0} l_{0}}\right)$ is nonzero in $H^{n-l_{0}+2}(X, \mathbb{Q})$. Thus $\partial: \pi_{l_{0}}\left(M_{f_{n} \circ e}\left(X, X_{n}\right)\right) \rightarrow \pi_{l_{0}-1}\left(M_{0}\left(X, K\left(V_{n+1}, n+1\right)\right)\right)$ is nontrivial.

\section{The Rational homotopy of $M_{1}(X, X)$}

The space $M_{1}(X, X)$, often written $G_{1}(X)$, is an $H$-space with multiplication defined by composition of maps. Its classifying space $B_{G_{1}(X)}$ (see [5]), is the universal cover of the classifying space, $B_{G(X)}$, for Hurewicz fibrations with fibre the homotopy type of $X$, as constructed by Stasheff [17] and Allaud [1]. Determining the rational homotopy type of $B_{G_{1}(X)}$ is an important and, in general, difficult problem. (See [18, pp. 313-314] for a description of the Quillen minimal model of $B_{G_{1}(X)}$ and [21] for an application of this model.) On the other hand, since $M_{1}(X, X)$ is an $H$-space it has the rational homotopy type of a product of rational Eilenberg-Mac Lane spaces. Thus determining the rational homotopy type of $M_{1}(X, X)$ is equivalent to computing its rational homotopy groups. In [16], we use Lemma 4.4 to compute the rational homotopy groups of $M_{1}(X, X)$ (and thus, by degree shifting, the rational homotopy groups of $\left.B_{G_{1}(X)}\right)$ for spaces $X$ whose rational cohomology is a tensor product of free algebras and truncated polynomial algebras-a class of spaces which includes products of spheres, projective spaces and Eilenberg-Mac Lane spaces. To indicate the method, we prove

Theorem 2. Let $X=S^{r_{1}} \times \cdots \times S^{r_{k}} \times S^{t_{1}} \times \cdots \times S^{t_{l}}$, where the $r_{i}$ are even, the $t_{i}$ odd and $r_{i}, t_{i}>1$. Then the rationalization of $M_{1}(X, X)$ is given by

$$
\left(M_{1}(X, X)\right)_{0}=\left(\prod_{i=1}^{k} \prod_{p=1}^{2 r_{i}-1} K\left(\mathbb{Q}^{b_{p}^{i}}, p\right)\right) \times\left(\prod_{i=1}^{l} \prod_{p=1}^{t_{i}} K\left(H^{t_{i}-p}(X, \mathbb{Q}), p\right)\right),
$$

where $b_{p}^{i}=\operatorname{dim}_{\mathbb{Q}}\left(H^{2 r_{i}-p-1}(X, \mathbb{Q})\right)-\operatorname{dim}_{\mathbb{Q}}\left(H^{r_{i}-p-1}(X, \mathbb{Q})\right)$.

Proof. To begin, observe that

$$
M_{1}(X, X) \approx\left(\prod_{i=1}^{k} M_{p_{i}}\left(X, S^{r_{i}}\right)\right) \times\left(\prod_{i=1}^{l} M_{q_{i}}\left(X, S^{t_{i}}\right)\right),
$$

where $p_{i}: X \rightarrow S^{r_{i}}$ and $q_{i}: X \rightarrow S^{t_{i}}$ are the projections. Since $t_{i}$ is odd, $\left(S^{t_{i}}\right)_{0}=K\left(\mathbb{Q}, t_{i}\right)$. It follows from Proposition 2.1 and Thom's result (3.1) that

$$
\left(M_{q_{i}}\left(X, S^{t_{i}}\right)\right)_{0}=\prod_{p=1}^{t_{i}} K\left(H^{t_{i}-p}(X, \mathbb{Q}), p\right) .
$$


It remains then to take $n=r_{i}$ to be even and compute the rational homotopy groups of $M_{p}\left(X, S^{n}\right)$, where $p=p_{i}: X \rightarrow S^{n}$ is the projection.

Let $e: S^{n} \rightarrow\left(S^{n}\right)_{0}$ be the rationalization of $S^{n}$. Let $\pi=e \circ p: X \rightarrow\left(S^{n}\right)_{0}$. Then, by Proposition 2.1, we have that $\left(M_{p}\left(X, S^{n}\right)\right)_{0} \simeq M_{\pi}\left(X,\left(S^{n}\right)_{0}\right)$. Now the Postnikov decompositon of $\left(S^{n}\right)_{0}$ is of the form

$$
K(\mathbb{Q}, 2 n-1) \hookrightarrow\left(S^{n}\right)_{0} \stackrel{q}{\rightarrow} K(\mathbb{Q}, n),
$$

with $k$-invariant $k_{2 n}=\imath_{n}^{2} \in H^{2 n}(K(\mathbb{Q}, n), \mathbb{Q})$ where $\imath_{n} \in H^{n}(K(\mathbb{Q}, n), \mathbb{Q})$ is the fundamental class. Accordingly, we have a fibre sequence of the form

$$
M_{0}(X, K(\mathbb{Q}, 2 n-1)) \rightarrow M_{\pi}\left(X,\left(S^{n}\right)_{0}\right) \stackrel{q}{\rightarrow} M_{q \circ \pi}(X, K(\mathbb{Q}, n)) .
$$

By Lemma 4.4, the boundary homomorphism

$$
\partial: \pi_{p}\left(M_{q \circ \pi}(X, K(\mathbb{Q}, n))\right) \rightarrow \pi_{p-1}\left(M_{0}(X, K(\mathbb{Q}, 2 n-1))\right),
$$

identified as in (4.3), is given by

$$
\partial\left(a_{n-p} \otimes l_{p}\right)=2 a_{n-p} x_{n} \in H^{2 n-p}(X, \mathbb{Q}),
$$

where $x_{n}=(q \circ \pi)^{*}\left(l_{n}\right) \in H^{n}(X, \mathbb{Q})$ and $a_{n-p} \in H^{n-p}(X, \mathbb{Q})$. Now multiplication by $x_{n}$ gives a linear injection of $H^{n-p}(X, \mathbb{Q})$ into $H^{2 n-p}(X, \mathbb{Q})$. (Note that $x_{n} \in H^{n}(X, \mathbb{Q})$ corresponds to a nontrivial element of $H^{n}\left(S^{n}, \mathbb{Q}\right)$ viewed in $H^{n}(X, \mathbb{Q})$.) Thus $\partial$ is injective for all $p>0$. Since the homotopy groups of $M_{0}(X, K(\mathbb{Q}, 2 n-1))$ and $M_{q \circ \pi}(X, K(\mathbb{Q}, n))$ are given by Thom's isomorphism (3.1) the result now follows from a dimension count.

\section{6. $M_{0}(X, X)_{*}$ AS $H$-SPACE}

In [3], Brown gives an example of spaces $X$ and $Y$ with $X$ not a suspension and $Y$ not an $H$-space such that the space of based inessential maps, $M_{0}(X, Y)_{*}$, has the structure of $H$-space. The following lemma provides more examples of this phenomenon:

Lemma 6.1. Let $X$ be a simply connected complex with only two nonvanishing, finitely generated homotopy groups, say $\pi_{n}(X)$ and $\pi_{m}(X)$, with $1<n<m$. Then

$$
M_{0}(X, X)_{*}=\prod_{i=1}^{m-1} K\left(H^{m-i}\left(X, \pi_{m}(X)\right), i\right) .
$$

Proof. If $Y$ is an $H$-space and $X$ is any complex, define $\Phi: Y \times M_{0}(X, Y)_{*} \rightarrow$ $M_{0}(X, Y)$ by $\Phi(y, f)(x)=y \cdot f(x)$, where the product is taken in $Y$. We claim that $\Phi$ is a weak equivalence. To prove this, we consider the evaluation fibration,

$$
M_{0}(X, Y)_{*} \hookrightarrow M_{0}(X, Y) \stackrel{\rho}{\rightarrow} Y,
$$

where $\rho$ is the map which evaluates a function in $M_{0}(X, Y)$ on the basepoint of $X$. It is easy to see that $\Phi$ is a fibre preserving map from the trivial fibration over $Y$ with fibre $M_{0}(X, Y)_{*}$ to the evaluation fibration. Moreover, $\Phi$ induces the identity map on the fibre over the basepoint of $Y$ and a homotopy equivalence on $Y$. Our claim follows from the five-lemma. 
An easy consequence of this fact and Thom's result (3.1) is that given a $K(\pi, n)$ with $n \geq 1$ and $\pi$ abelian and any complex $X$, we have a weak equivalence

$$
M_{0}(X, K(\pi, n))_{*} \simeq_{w} \prod_{i=1}^{n-1} K\left(H^{n-i}(X, \pi), i\right) .
$$

To prove the lemma, we view $\mathrm{X}$ as the total space of the principal fibration $K\left(\pi_{m}(X), m\right) \hookrightarrow X \stackrel{p}{\rightarrow} K\left(\pi_{n}(X), n\right)$, and observe that there then exists a fibre sequence of based mapping spaces of the form

$$
M_{0}\left(X, K\left(\pi_{m}(X), m\right)\right)_{*} \stackrel{i}{\rightarrow} M_{0}(X, X)_{*} \stackrel{p}{\rightarrow} M_{0}\left(X, K\left(\pi_{n}(X), n\right)\right)_{*} .
$$

Since $X$ is $n-1$ connected $n>1, H^{q}\left(X, \pi_{n}(X)\right)=0$, for all $0<q<$ $n$. By (6.2), $M_{0}\left(X, K\left(\pi_{n}(X), n\right)\right)_{*}$ is weakly contractible and so $i$ is a weak equivalence. By Proposition 2.1, $i$ is a map between $\mathrm{CW}$ complexes and so the result follows from (6.2) and Whitehead's theorem.

\section{A Result of Haefliger}

Up to now we have concerned ourselves primarily with computing rational homotopy groups of function spaces, for which Thom's approach of using Postnikov towers is well-suited. Our proof of Theorem 3, however, involves determining the rational homotopy type of a component of a function space. As Thom's work in [20] indicates, an effective approach to this problem is to analyze the relevant evaluation maps. In this section, we recall a result of Haefliger and apply it to deduce a characterization of certain evaluation maps.

Let $X$ and $Z$ be connected complexes, $V_{n}$ a rational vector space, and $n>0$. In [7, §2.1], Haefliger characterizes maps of the form

$$
\varphi: Z \rightarrow M\left(X, K\left(V_{n}, n\right)\right),
$$

as follows. By the exponential law, $\varphi$ is adjoint to a map $\tilde{\varphi}: X \times Z \rightarrow$ $K\left(V_{n}, n\right)$. By Thom's result,

$$
M\left(X, K\left(V_{n}, n\right)\right)=\prod_{i=0}^{n} K_{i}
$$

where $K_{i}=K\left(H^{n-i}\left(X, V_{n}\right), i\right)$. Let $\varphi_{i}: Z \rightarrow K_{i}$ denote $\varphi$ composed with the $i$ th projection. Observe that

$$
H^{i}\left(K_{i}, \mathbb{Q}\right) \cong H_{n-i}(X, \mathbb{Q}) \otimes V_{n}^{*}
$$

where we view $H_{n-i}(X, \mathbb{Q})$ as the dual space of $H^{n-i}(X, \mathbb{Q})$ and $V_{n}^{*}=$ $H^{n}\left(K\left(V_{n}, n\right), \mathbb{Q}\right)$. According to Haefliger, we have

Lemma 7.1 (Haefliger). Given $a^{\prime} \otimes v \in H_{n-i}(X, \mathbb{Q}) \otimes V_{n}^{*} \cong H^{n-i}\left(K_{i}, \mathbb{Q}\right)$ then

$$
\varphi_{i}^{*}\left(a^{\prime} \otimes v\right)=a^{\prime} \cap \tilde{\varphi}^{*}(v) \quad \text { where } a^{\prime} \cap(a \otimes b)=a^{\prime}(a) b,
$$

for $a \otimes b \in H^{*}(X, \mathbb{Q}) \otimes H^{*}(Z, \mathbb{Q}) \cong H^{*}(X \times Z, \mathbb{Q})$. 
We apply Haefliger's lemma to characterize the evaluation map

$$
\varepsilon: X \times M\left(X, K\left(V_{n}, n\right)\right) \rightarrow K\left(V_{n}, n\right),
$$

defined by

$$
\varepsilon(x, f)=f(x),
$$

and its restriction to a component $M_{f}\left(X, K\left(V_{n}, n\right)\right)$ of $M\left(X, K\left(V_{n}, n\right)\right)$ which we denote by

$$
\varepsilon_{f}: X \times M_{f}\left(X, K\left(V_{n}, n\right)\right) \rightarrow K\left(V_{n}, n\right) .
$$

Choose an additive basis $\left\{a_{i j} \mid i=0, \ldots, n ; j=1, \ldots, \beta_{i}\right\}$ of $H^{\leq n}(X, \mathbb{Q})$ where $a_{i j} \in H^{i}(X, \mathbb{Q})$ and $\beta_{i}=\operatorname{dim}_{\mathbb{Q}}\left(H^{i}(X, \mathbb{Q})\right)$. Let $b_{i j} \in H_{i}(X, \mathbb{Q}), j=$ $1, \ldots, \beta_{i}$ be the basis dual to the $a_{i j}$. Let $a_{0}=a_{0,1}=1 \in H^{0}(X, \mathbb{Q})$ and let $b_{0}=b_{0,1}=1^{\prime}$ be its dual. Let $v \in V_{n}^{*}=H^{n}\left(K\left(V_{n}, n\right), \mathbb{Q}\right)$. Observe that

$$
\begin{aligned}
\varepsilon^{*}(v) & \in H^{n}\left(X \times M\left(X, K\left(V_{n}, n\right)\right), \mathbb{Q}\right) \\
& \cong \bigoplus_{i=0}^{n} H^{n-i}(X, \mathbb{Q}) \otimes H^{i}\left(M\left(X, K\left(V_{n}, n\right)\right), \mathbb{Q}\right) \\
& \cong \bigoplus_{i=0}^{n} H^{n-i}(X, \mathbb{Q}) \otimes H^{i}\left(\prod_{j=0}^{n} K_{j}, \mathbb{Q}\right) .
\end{aligned}
$$

Since $M_{f}\left(X, K\left(V_{n}, n\right)\right.$ is connected,

$$
\varepsilon_{f}^{*}(v) \in \bigoplus_{i=0}^{n} H^{n-i}(X, \mathbb{Q}) \otimes H^{i}\left(\prod_{j=1}^{n} K_{j}, \mathbb{Q}\right)
$$

Recalling that $H^{i}\left(K_{i}, \mathbb{Q}\right)=H^{i}\left(K\left(H^{n-i}\left(X, V_{n}\right), i\right), \mathbb{Q}\right) \cong H_{n-i}(X, \mathbb{Q}) \otimes V_{n}^{*}$, we prove

Lemma 7.2 (cf. [15, pp. 323-324]).

$$
\begin{aligned}
& \varepsilon^{*}(v)=\sum_{i=0}^{n} \sum_{j=1}^{\beta_{i}} a_{i j} \otimes\left(b_{i j} \otimes v\right), \\
& \varepsilon_{f}^{*}(v)=\sum_{i=0}^{n-1} \sum_{j=1}^{\beta_{i}} a_{i j} \otimes\left(b_{i j} \otimes v\right)+f^{*}(v) \otimes 1 .
\end{aligned}
$$

Proof. We deduce (i) from Haefliger's result (Lemma 7.1). Note that the adjoint of $\varepsilon$

$$
\varphi: M\left(X, K\left(V_{n}, n\right)\right) \rightarrow M\left(X, K\left(V_{n}, n\right)\right)
$$

is just the identity map. Let $\varphi_{i}: M\left(X, K\left(V_{n}, n\right)\right) \rightarrow K_{i}$ be the projection. Then, by Lemma 7.1, if $b_{n-i, j} \otimes v \in H_{n-i}(X, \mathbb{Q}) \otimes V_{n}^{*}=H^{i}\left(K_{i}, \mathbb{Q}\right)$,

$$
\varphi_{i}^{*}\left(b_{n-i, j} \otimes v\right)=b_{n-i, j} \cap \varepsilon^{*}(v) .
$$


On the other hand, $\varphi_{i}^{*}\left(b_{n-i, j} \otimes v\right)=b_{n-i, j} \otimes v \in H^{i}\left(M\left(X, K\left(V_{n}, n\right)\right), \mathbb{Q}\right)$, since $\varphi$ is the identity. Since $\left\{a_{i j}\right\}$ is an additive basis for $H^{\leq n}(X, \mathbb{Q})$ and

$$
\varepsilon^{*}(v) \in \bigoplus_{i=0}^{n} H^{n-i}(X, \mathbb{Q}) \otimes H^{i}\left(M\left(X, K\left(V_{n}, n\right)\right), \mathbb{Q}\right),
$$

we may write

$$
\varepsilon^{*}(v)=\sum_{i=0}^{n} \sum_{j=1}^{\beta_{i}} a_{n-i, j} \otimes c_{i j}
$$

for some $c_{i j} \in H^{i}\left(M\left(X, K\left(V_{n}, n\right)\right), \mathbb{Q}\right)$. But now we have

$$
\begin{aligned}
b_{n-i, j} \otimes v & =\varphi_{i}^{*}\left(b_{n-i, j} \otimes v\right)=b_{n-i, j} \cap \varepsilon^{*}(v) \\
& =b_{n-i, j} \cap\left(a_{n-i, j} \otimes c_{i j}\right)=c_{i j} .
\end{aligned}
$$

This proves part (i).

For (ii) we observe that the composition

$$
X \hookrightarrow X \times M_{f}\left(X, K\left(V_{n}, n\right)\right) \stackrel{\varepsilon_{f}}{\rightarrow} K\left(V_{n}, n\right)
$$

is just the map $f: X \rightarrow K\left(V_{n}, n\right)$, which fact, together with (i), implies the result.

\section{Proof of Theorem 3}

Theorem 3. Let $X$ be a simply connected complex with finitely many, finitely generated homotopy groups whose rationalization is a two-stage Postnikov system. Then

$$
M_{0}(X, X) \simeq_{\mathbb{Q}} X \times M_{0}(X, X)_{*} .
$$

Proof. Our technique will be to construct the minimal model for $M_{0}(X, X)$. We follow the approach of Møller and Raussen [15].

We assume that the nontrivial rational homotopy groups of $X$ occur in dimensions $n$ and $m$ with $1<n<m$. Put $V_{n}=\pi_{n}(X) \otimes \mathbb{Q}$ and $V_{m}=$ $\pi_{m}(X) \otimes \mathbb{Q}$. Then the rationalization $X_{0}$ of $X$ is given by the pullback diagram

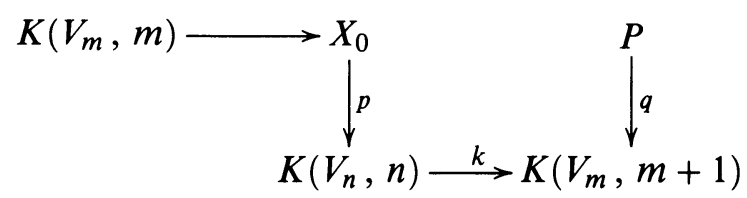

where $q: P \rightarrow K\left(V_{m}, m+1\right)$ is the path/loop fibration and where $k \in$ $H^{m+1}\left(K\left(V_{n}, n\right), \mathbb{Q}\right)$ is the rational $k$-invariant of $X$. If $k$ is zero than $X_{0}$ is an $H$-space. By the first paragraph of Lemma 6.1, we have that

$$
M_{0}\left(X, X_{0}\right) \simeq X_{0} \times M_{0}\left(X, X_{0}\right)_{*} \text {. }
$$

The result now follows, in this case, from Proposition 2.1 and we may assume then that $k$ is nontrivial. 
By [4, Lemma 3.2], the rational Postnikov tower of $X$ determines the minimal model, $\left(\mathscr{M}^{*}(X), d_{X}\right)$, of $X$. Specifically, $\left(\mathscr{M}^{*}(X), d_{X}\right)$ is of the form

$$
\mathscr{M}^{*}(X)=\Lambda_{n}\left(V_{n}^{*}\right) \otimes_{d_{X}} \Lambda_{m}\left(V_{m}^{*}\right) .
$$

Regarding the differential $d_{X}$, note that $d_{X}$ may be viewed as a linear map $d_{X}: V_{m}^{*} \rightarrow H^{m+1}\left(K\left(V_{n}, n\right), \mathbb{Q}\right)$ and so as an element of $H^{m+1}\left(K\left(V_{n}, n\right), V_{m}\right)$. According to the lemma, the differential coincides with the rational $k$-invariant $k$ of $X$.

Since $k$ is nontrivial, $H^{m+1}\left(K\left(V_{n}, n\right), \mathbb{Q}\right) \neq 0$. It follows that $n \mid m+1$. Since $n>1$, we cannot have that $n$ divides $m$ also. Given the form of $\left(\mathscr{M}^{*}(X), d_{X}\right)$ we conclude that

$$
H^{m}(X, \mathbb{Q}) \cong \operatorname{ker}\left\{d_{X}: V_{m}^{*} \rightarrow H^{m+1}\left(K\left(V_{n}, n\right), \mathbb{Q}\right)\right\} .
$$

We assume, at first, that $d_{X}$ is injective so that $H^{m}(X, \mathbb{Q})=0$.

The fibration $p: X_{0} \rightarrow K\left(V_{n}, n\right)$ induces a fibration on free mapping spaces

$$
\underline{p}: M_{0}\left(X, X_{0}\right) \rightarrow M_{0}\left(X, K\left(V_{n}, n\right)\right) .
$$

Since, under our current assumptions, $\left[X, K\left(V_{m}, m\right)\right] \cong H^{m}\left(X, V_{m}\right)=0$, the mapping space $M\left(X, K\left(V_{m}, m\right)\right)$ is connected and it follows that the the fibre of $\underline{p}$ is $M\left(X, K\left(V_{m}, m\right)\right)$. By Thom's result, the rational cohomology of $M\left(X, K\left(V_{m}, m\right)\right)$ is free as a graded algebra. We wish to apply the Hirsch Lemma [4, Lemma 3.1] to the fibration $\underline{p}$ and so obtain a model for $M_{0}\left(X, X_{0}\right)$. To apply the lemma, however, we must first show that the rational cohomology of $M\left(X, K\left(V_{m}, m\right)\right)$ is transgressive (see [4, p. 257]) with respect to the fibration $\underline{p}$.

To this end, consider the fibration on mapping spaces

$$
\underline{q}: M(X, P) \rightarrow M_{0}\left(X, K\left(V_{m}, m+1\right)\right),
$$

induced by $q: P \rightarrow K\left(V_{m}, m+1\right)$, with fibre $M\left(X, K\left(V_{m}, m\right)\right)$. From the exponential law and the definition of $P$ it is clear that $M(X, P)$ is homeomorphic to the path space on $M_{0}\left(X, K\left(V_{m}, m+1\right)\right)$. Thus $\underline{q}$ is just the path/loop fibration over a product of Eilenberg-Mac Lane spaces. As such, the rational cohomology of $M\left(X, K\left(V_{m}, m\right)\right)$ is easily seen to be transgressive with respect to $\underline{q}$. To show that it is transgressive with respect to $\underline{p}$, we show that the fibration $\underline{p}: M_{0}\left(X, X_{0}\right) \rightarrow M_{0}\left(X, K\left(V_{n}, n\right)\right)$ is the pullback of $\underline{q}$ via the map

$$
\underline{k}: M_{0}\left(X, K\left(V_{n}, n\right)\right) \rightarrow M_{0}\left(X, K\left(V_{m}, m+1\right)\right) \text {. }
$$

Let $E$ denote the total space of the pullback of $\underline{q}$ by $\underline{k}$. Then it is easy to check that

$$
E=\left\{f: X \rightarrow X_{0} \mid p \circ f \simeq *\right\} .
$$

Thus $M_{0}\left(X, X_{0}\right)$ is a full component of $E$. To show equality, we consider the exact sequence of sets of homotopy classes of maps corresponding to the fibration $K\left(V_{m}, m\right) \hookrightarrow X_{0} \stackrel{p}{\rightarrow} K\left(V_{n}, n\right)$, a portion of which is given as

$$
\left[X, K\left(V_{m}, m\right)\right] \rightarrow\left[X, X_{0}\right] \stackrel{p_{*}}{\rightarrow}\left[X, K\left(V_{n}, n\right)\right] .
$$

Note that, by our assumption, $\left[X, K\left(V_{m}, m\right)\right]=H^{m}\left(X, V_{m}\right)=0$. Thus $p_{*}$ is injective as a map of sets and it follows that $E=M_{0}\left(X, X_{0}\right)$. 
We can now apply the Hirsch Lemma to obtain a model for $M_{0}\left(X, X_{0}\right)$. By Thom's result, $M\left(X, K\left(V_{m}, m\right)\right)$ and $M_{0}\left(X, K\left(V_{n}, n\right)\right)$ are products of Eilenberg-Mac Lane spaces. Thus the minimal models of these spaces are simply their rational cohomology algebras with trivial differentials. By the Hirsch Lemma, a model $\left(\mathscr{A}^{*}\left(M_{0}\left(X, X_{0}\right)\right), \delta\right)$ for $M_{0}\left(X, X_{0}\right)$ is given in the form

$$
\mathscr{A}^{*}\left(M_{0}\left(X, X_{0}\right)\right)=H^{*}\left(M_{0}\left(X, K\left(V_{n}, n\right)\right), \mathbb{Q}\right) \otimes_{\delta} H^{*}\left(M\left(X, K\left(V_{m}, m\right)\right), \mathbb{Q}\right)
$$

where the differential $\delta$ is just the transgression of the fibration $\underline{p}$. We seek to determine the differential explicitly.

Write $M_{0}\left(X, K\left(V_{m}, m+1\right)\right)=\prod_{i=1}^{m+1} K_{i}$ where $K_{i}=K\left(H^{m-i+1}\left(X, V_{m}\right), i\right)$. Let $\underline{k}_{i}: M_{0}\left(X, K\left(V_{n}, n\right)\right) \rightarrow K_{i}$ denote $\underline{k}$ composed with the projection. Since $\underline{p}$ is the pullback of $\underline{q}$ by $\underline{k}$, the $\underline{k}_{i}$ are the $k$-invariants for $M_{0}\left(X, X_{0}\right)$, and, as such, they determine the transgression of $\underline{p}$.

To be more explicit, write

$$
H^{*}\left(M\left(X, K\left(V_{m}, m\right)\right), \mathbb{Q}\right)=\prod_{i=1}^{m} \Lambda_{i}\left(H_{m-i}\left(X, V_{m}\right)\right)
$$

where we identify $H_{m-i}\left(X, V_{m}\right)=H^{m-i}\left(K\left(H^{m-i}\left(X, V_{m}\right), i\right), \mathbb{Q}\right)$. Also, let us write $H_{m-i}\left(X, V_{m}\right)=H_{m-i}(X, \mathbb{Q}) \otimes V_{m}^{*}$. Then given

$$
b_{m-i, j} \otimes v \in H_{m-i}(X, \mathbb{Q}) \otimes V_{m}^{*}=\operatorname{Vect}_{i}\left(H^{*}\left(M\left(X, K\left(V_{m}, m\right)\right), \mathbb{Q}\right)\right),
$$

we note that $b_{m-i, j} \otimes v \in H_{m-i}\left(X, V_{m}\right)=H^{i+1}\left(K\left(H^{m-i}\left(X, V_{m}\right), i+1\right), \mathbb{Q}\right)=$ $H^{i+1}\left(K_{i+1}, \mathbb{Q}\right)$. Thus $\underline{k}_{i+1}^{*}\left(b_{m-i, j} \otimes v\right) \in H^{i+1}\left(M_{0}\left(X, K\left(V_{n}, n\right)\right), \mathbb{Q}\right)$. By the Hirsch Lemma, the differential

$$
\delta: \operatorname{Vect}_{i}\left(H^{*}\left(M\left(X, K\left(V_{m}, m\right)\right), \mathbb{Q}\right)\right) \rightarrow H^{i+1}\left(M_{0}\left(X, K\left(V_{n}, n\right)\right), \mathbb{Q}\right)
$$

is given by the equation

$$
\delta\left(b_{m-i, j} \otimes v\right)=\underline{k}_{i+1}^{*}\left(b_{m-i, j} \otimes v\right) .
$$

We now apply Haefliger's result (Lemma 7.1). Note that $\underline{k}$ is adjoint to the map $g=k \circ \varepsilon_{0}$ where $\varepsilon_{0}: X \times M_{0}\left(X, K\left(V_{n}, n\right)\right) \rightarrow K\left(V_{n}, n\right)$ is the evaluation map restricted to the component of the constant map in $M\left(X, K\left(V_{n}, n\right)\right)$. By (8.1) and Lemma 7.1,

$$
\begin{aligned}
\delta\left(b_{m-i, j} \otimes v\right) & =\underline{k}_{i+1}^{*}\left(b_{m-i, j} \otimes v\right)=b_{m-i, j} \cap g^{*}(v) \\
& =b_{m-i, j} \cap \varepsilon_{0}^{*}\left(k^{*}(v)\right),
\end{aligned}
$$

Let $x_{1}, \ldots, x_{s}$ be a basis for $H^{n}\left(K\left(V_{n}, n\right), \mathbb{Q}\right)$. Let $v_{1}, \ldots, v_{t}$ be a basis for $V_{m}^{*}$. Recall $k^{*}=d_{X}: V_{m}^{*} \rightarrow H^{m+1}\left(K\left(V_{n}, n\right), \mathbb{Q}\right)$, where $d_{X}$ is the differential for the minimal model of $X$. As usual, write $k^{*}\left(v_{l}\right)=p_{l}\left(x_{1}, \ldots, x_{s}\right)$ where $p_{l}$ is some homogeneous polynomial in $s$ variables of appropriate degree. Now $H^{j}(X, \mathbb{Q})=0$ for $0<j<n$. By Lemma 7.2 then, the evaluation map $\varepsilon_{0}: X \times M_{0}\left(X, K\left(V_{n}, n\right)\right) \rightarrow K\left(V_{n}, n\right)$ is given simply by the equation

$$
\varepsilon_{0}^{*}\left(x_{i}\right)=1 \otimes b_{0} \otimes x_{i}
$$


where we recall $b_{0} \in H_{0}(X, \mathbb{Q})$ is the dual vector to $1 \in H^{0}(X, \mathbb{Q})$. We see then that, for $v_{l} \in V_{m}^{*}$, we have

$$
\begin{aligned}
\varepsilon_{0}^{*}\left(k^{*}\left(v_{l}\right)\right) & =\varepsilon_{0}^{*}\left(p_{l}\left(x_{1}, \ldots, x_{s}\right)\right) \\
& =p_{l}\left(1 \otimes b_{0} \otimes x_{1}, \ldots, 1 \otimes b_{0} \otimes x_{s}\right) \\
& =1 \otimes p_{l}\left(b_{0} \otimes x_{1}, \ldots, b_{0} \otimes x_{s}\right) .
\end{aligned}
$$

Combining (8.2) and (8.3), we obtain the following description of the differential $\delta$. Given $b_{m-i, j} \otimes v_{l} \in \operatorname{Vect}_{i}\left(H^{*}\left(M\left(X, K\left(V_{m}, m\right)\right)\right)\right)$ for $0<i<m$ we have

$$
\begin{aligned}
\delta\left(b_{m-i, j} \otimes v_{l}\right) & =b_{m-i, j} \cap \varepsilon_{0}^{*}\left(k^{*}\left(v_{l}\right)\right)=b_{m-i, j} \cap\left(1 \otimes p_{l}\left(b_{0} \otimes x_{1}, \ldots, b_{0} \otimes x_{s}\right)\right) \\
& =b_{m-i, j}(1) \cdot p_{l}\left(b_{0} \otimes x_{1}, \ldots, b_{0} \otimes x_{s}\right)=0,
\end{aligned}
$$

since $b_{m-i, j}(1)=0$ for $0<i<m$. Similarly, on the basis $\left\{b_{0} \otimes v_{1}, \ldots, b_{0} \otimes\right.$ $\left.v_{t}\right\}$ of $\operatorname{Vect}_{m}\left(H^{*}\left(M\left(X, K\left(V_{m}, m\right)\right)\right)\right)$ we see that $\delta$ is given by

$$
\delta\left(b_{0} \otimes v_{l}\right)=p_{l}\left(b_{0} \otimes x_{1}, \ldots, b_{0} \otimes x_{s}\right) .
$$

We conclude that the model $\left(\mathscr{A}^{*}\left(M_{0}\left(X, X_{0}\right)\right), \delta\right)$ may be written in the form

$$
\mathscr{A}^{*}\left(M_{0}\left(X, X_{0}\right)\right)=\left(\Lambda_{n}\left(V_{n}^{*}\right) \otimes_{d_{X}} \Lambda_{m}\left(V_{m}^{*}\right)\right) \otimes\left(\bigotimes_{i=1}^{m-1} \Lambda_{i}\left(H_{m-i}\left(X, V_{m}\right)\right)\right),
$$

where $d_{X}$ is just the differential of the minimal model of $X$. It follows that $\mathscr{A}^{*}\left(M_{0}\left(X, X_{0}\right)\right)$ is, in fact, the minimal model for $M_{0}\left(X, X_{0}\right)$. Since the minimal model of $X$ is a factor in the minimal model of $M_{0}\left(X, X_{0}\right)$, by uniqueness, we obtain

$$
M_{0}\left(X, X_{0}\right) \simeq_{\mathbb{Q}} X \times \prod_{i=1}^{m-1} K\left(H^{m-i}\left(X, V_{m}\right), i\right) .
$$

Now, using the proof of Lemma 6.1 and Proposition 2.1, it is easy to see that

$$
\left(M_{0}(X, X)_{*}\right)_{0}=\prod_{i=1}^{m-1} K\left(H^{m-i}\left(X, V_{m}\right), i\right) .
$$

Thus, since $M_{0}(X, X) \simeq_{\mathbb{Q}} M_{0}\left(X, X_{0}\right)$, the theorem is proved in the case where $d_{X}: V_{m}^{*} \rightarrow H^{m+1}\left(K\left(V_{n}, n\right), \mathbb{Q}\right)$ is injective.

For the general case, we write $V_{m}^{*}=W^{*} \oplus V^{*}$ where $V^{*}=\operatorname{ker}\left\{d_{X}: V_{m}^{*} \rightarrow\right.$ $\left.H^{m+1}\left(K\left(V_{n}, n\right), \mathbb{Q}\right)\right\}$. Then we may decompose $X_{0}$ as the product $X_{0}=Y_{0} \times$ $K(V, m)$ where $Y_{0}$ is a rational two-stage Postnikov system with injective $k$-invariant. Let $Y$ be a two-stage Postnikov system with finitely generated homotopy groups whose rationalization is $Y_{0}$. Then, by the above, we have that $M_{0}(Y, Y) \simeq_{\mathbb{Q}} Y \times M_{0}(Y, Y)_{*}$.

Using Proposition 2.1 and an induction on the height of a Postnikov tower, it is easy to show that, given a simply connected complex $Z$ with finitely many, 
finitely generated homotopy groups, there is a weak equivalence

$$
\left(M_{0}(Z, Z)\right)_{0} \simeq{ }_{w} M_{0}\left(Z_{0}, Z_{0}\right)
$$

where $\left(M_{0}(Z, Z)\right)_{0}$ is the rationalization of $M_{0}(Z, Z)$. The analogous result holds for the space of based inessential maps. Applying (8.4) to our rational decomposition of $M_{0}(Y, Y)$ we get

$$
M_{0}\left(Y_{0}, Y_{0}\right) \simeq_{w} Y_{0} \times M_{0}\left(Y_{0}, Y_{0}\right)_{*}
$$

From (8.4), (8.5) and the exponential law we obtain the following chain of equivalences:

$$
\begin{aligned}
\left(M_{0}(X, X)\right)_{0} \simeq & M_{0}\left(Y_{0} \times K(V, m), Y_{0} \times K(V, m)\right) \\
\approx & M_{0}\left(Y_{0} \times K(V, m), Y_{0}\right) \times M_{0}\left(Y_{0} \times K(V, m), K(V, m)\right) \\
\approx & M_{0}\left(K(V, m), M_{0}\left(Y_{0}, Y_{0}\right)\right) \times M_{0}\left(X_{0}, K(V, m)\right) \\
\simeq & M_{0}\left(K(V, m), Y_{0} \times M_{0}\left(Y_{0}, Y_{0}\right)_{*}\right) \times M_{0}\left(X_{0}, K(V, m)\right) \\
\approx & M_{0}\left(K(V, m), Y_{0}\right) \times M_{0}\left(K(V, m), M_{0}\left(Y_{0}, Y_{0}\right)_{*}\right) \\
& \times M_{0}\left(X_{0}, K(V, m)\right) .
\end{aligned}
$$

Now, since $Y_{0}$ is $m+1$ coconnected and $K(V, m)$ is $m-1$-connected, we see that, for $p>0, \quad \pi_{p}\left(M_{0}\left(K(V, m), Y_{0}\right)_{*}\right) \cong\left[K\left(V_{m}, m\right), \Omega^{p}\left(Y_{0}\right)\right]=0$, so that $M_{0}\left(K(V, m), Y_{0}\right)_{*}$ is weakly contractible. From the evaluation fibration (see Lemma 6.1)

$$
M_{0}\left(K(V, m), Y_{0}\right)_{*} \hookrightarrow M_{0}\left(K(V, m), Y_{0}\right) \stackrel{\rho}{\rightarrow} Y_{0}
$$

we obtain that

$$
M_{0}\left(K(V, m), Y_{0}\right) \simeq_{w} Y_{0} .
$$

From Lemma 6.1 and (8.4), we have

$$
M_{0}\left(Y_{0}, Y_{0}\right)_{*} \simeq_{w} \prod_{i=1}^{m-1} K\left(H^{m-i}\left(Y_{0}, W\right), i\right)
$$

so that, by Thom's result (3.1),

$$
M_{0}\left(K(V, m), M_{0}\left(Y_{0}, Y_{0}\right)_{*}\right) \simeq_{w} \prod_{i=1}^{m-1} K\left(H^{m-i}\left(Y_{0}, W\right), i\right)
$$

Also, we have

$$
M_{0}\left(X_{0}, K(V, m)\right) \simeq_{w} \prod_{i=1}^{m} K\left(H^{m-i}(X, V), i\right) .
$$


Substituting (8.7), (8.8), and (8.9) into (8.6), we obtain

$$
\begin{aligned}
M_{0}(X, X) & \simeq_{\mathbb{Q}} Y_{0} \times \prod_{i=1}^{m-1} K\left(H^{m-i}\left(Y_{0}, W\right), i\right) \times \prod_{i=1}^{m} K\left(H^{m-i}(X, V), i\right) \\
& =Y_{0} \times K(V, m) \times \prod_{i=1}^{m-1} K\left(H^{m-i}\left(Y_{0}, W\right) \oplus H^{m-i}(X, V), i\right) \\
& \simeq_{\mathbb{Q}} X \times \prod_{i=1}^{m-1} K\left(H^{m-i}\left(X, V_{m}\right), i\right) \\
& \simeq_{\mathbb{Q}} X \times M_{0}(X, X)_{*},
\end{aligned}
$$

and the proof is complete.

Remark. Hansen [9, Corollary 3.5] proves that for $n \geq 8, n \neq 11,27$, and $n \not \equiv 15 \bmod 16, M_{0}\left(S^{n}, S^{n}\right)$ is not homotopy equivalent to $S^{n} \times M_{0}\left(S^{n}, S^{n}\right)_{*}$. Thus there is no analogue of Theorem 3 in ordinary homotopy theory.

\section{REFERENCES}

1. G. Allaud, On the classification of fibre spaces, Math. Z. 92 (1966), 110-125.

2. A. K. Bousfield and V. K. A. M. Gugenheim, On PL de Rham theory and rational homotopy type, Mem. Amer. Math. Soc. 8 (1976).

3. R. Brown, Two examples in homotopy theory, Proc. Cambridge Philos. Soc. 62 (1966), 575576.

4. P. Deligne, P. Griffiths, J. Morgan, and D. Sullivan, Real homotopy theory of Kähler manifolds, Invent. Math. 29 (1975), 245-274.

5. A. Dold and R. Lashof, Principal quasi-fibrations and fibre homotopy equivalence of bundles, Illinois J. Math. 3 (1959), 285-305.

6. H. Federer, $A$ study of function spaces by spectral sequences, Trans. Amer. Math. Soc. 82 (1956), 340-361.

7. A. Haefliger, Rational homotopy of the space of sections of a nilpotent bundle, Trans. Amer. Math. Soc. 273 (1982), 609-620.

8. V. L. Hansen, On spaces of maps of n-manifolds into the n-sphere, Trans. Amer. Math. Soc. 265 (1981), 273-281.

9. $\longrightarrow$ Decomposability of evaluation fibrations and the brace product operation of James, Compositio Math. 35 (1977), 83-89.

10. P. Hilton, G. Mislin, and J. Roitberg, Localization of nilpotent groups and spaces, Math. Stud., vol. 15, North-Holland, Amsterdam, 1975.

11. P. Hilton, G. Mislin, J. Roitberg, and R. Steiner, On free maps and free homotopies into nilpotent spaces, Lecture Notes in Math., vol. 673, Springer-Verlag, Berlin and New York, 1978.

12. P. J. Kahn, Some function spaces of CW type, Proc. Amer. Math. Soc. 90 (1984), 599-607.

13. J. Milnor, On spaces having the homotopy type of a CW complex, Trans. Amer. Math. Soc. 90 (1959), 272-280.

14. J. M. Møller, On spaces of maps between complex projective spaces, Proc. Amer. Math. Soc. 91 (1984), 471-476.

15. J. M. Møller and M. Raussen, Rational homotopy of spaces of maps into spheres and complex projective spaces, Trans. Amer. Math. Soc. 292 (1985), 721-732.

16. S. Smith, The rational homotopy of mapping spaces, Tech. Report, School of Math., University of Minnesota, Minneapolis, 1992. 
17. J. Stasheff, A classification theorem for fibre spaces, Topology 2 (1963), 239-246.

18. D. Sullivan, Infinitesimal computations in topology, Inst. Hautes Études Sci. Publ. Math. 47 (1977), 269-331.

19. D. Tanré, Homotopie rationelle: Modèles de Chen, Quillen, Sullivan, Lecture Notes in Math., vol. 1025, Springer-Verlag, Berlin and New York, 1983.

20. R. Thom, L'homologie des espaces fonctionelles, Colloq. Topologie Algébrique, Louvain 1956, pp. 29-39.

21. K. Yamaguchi, On the rational homotopy of $\operatorname{Map}\left(\mathbb{H} P^{m}, \mathbb{H} P^{n}\right)$, Kodai Math. J. 6 (1983), 279-288.

School of Mathematics, University of Minnesota, Minneapolis, Minnesota 55455

Current address: Department of Mathematics, Washington College, Chestertown, Maryland 21620

E-mail address: sam_smith.facultywashcoll.edu 